\title{
The control of attentional target selection in a colour/colour conjunction task
}

\author{
Nick Berggren $^{1}$ - Martin Eimer ${ }^{1}$ \\ Published online: 29 June 2016 \\ (C) The Psychonomic Society, Inc. 2016
}

Keywords Selective attention · Top-down control · Visual search $\cdot$ Event-related brain potentials $\cdot$ Feature-based attention $\cdot$ Feature integration $\cdot$ Object-based attention

\section{Introduction}

When searching for targets in visual scenes, top-down attentional control is necessary to detect and select task-relevant features and objects and to ignore irrelevant items. The allocation of attention can be guided by features known to be associated with target stimuli. For example, during search for a specific colour (e.g., red), attention will be rapidly biased towards red objects and not towards objects in different colours, even when these are perceptually salient (Folk \& Remington, 1998). The maintenance of known featural properties of target objects has been described as activating an attentional template (Desimone \& Duncan, 1995; Duncan \& Humphreys, 1989) or attentional control setting (Folk, Remington \& Johnston, 1992). These templates are believed to be initially represented in visual working memory but may be transferred to a long-term memory store when observers search for the same target features across a number of trials (Carlisle, Arita, Pardo, \& Woodman, 2011). Attentional templates can modulate neural activity in visual areas before stimuli being presented, such as increasing activation in colourselective versus motion-selective visual areas when anticipating a target in one dimension versus another (Shibata et al., 2008; see Eimer, 2014, 2015, for reviews). While attentional templates have been shown to bias attention towards a particular feature (e.g., red) or a feature dimension (e.g., colour; Folk \& Anderson, 2010; Müller, Heller, \& Ziegler, 1995; Töllner, Gramann, Müller, Kiss, \& Eimer, 2008), detecting a conjunctively defined target (e.g., a red square among blue squares and red circles) is typically less efficient than finding

\footnotetext{
Department of Psychological Sciences, Birkbeck College, University of London, Malet Street, London WC1E 7HX, UK
}

Nick Berggren

nbergg01@mail.bbk.ac.uk 
objects with a unique target-defining feature (Treisman \& Gelade, 1980). Indeed, these kinds of search are more akin to real-world scenes, where target objects are usually composed of multiple features. Thus, the question how target templates may operate beyond search for individual features is a key issue in understanding top-down attentional control.

The mechanisms that may be involved in search for targets defined by a feature conjunction have been described by a leading computational model of visual search (Guided Search; Wolfe, 1994; 2007). According to this model, individual feature channels analyse the properties of incoming sensory information from different dimensions in parallel across the visual field. Signals from target-defining features are weighted more strongly than signals from task-irrelevant features; these weighted signals are then combined on a spatiotopic saliency map, which controls where attention will be deployed. As a result, attention is most likely to be allocated first to objects that match the greatest number of targetdefining features, because these stimuli trigger the highest activation on the salience map. Guided Search assumes that the selection of particular objects in a search display operates in a serial fashion for one object at a time. At a subsequent stage of attentional processing where the identity of selected objects and their status as targets or nontargets is determined, multiple objects can be analysed in parallel (Wolfe, 2007). This hybrid model of attentional mechanisms during visual search, where an early serial object selection stage is followed by a parallel object identification stage, has been described in analogy to a carwash where cars enter one-by-one but multiple cars can be processed at any one time (Moore \& Wolfe, 2001).

According to Guided Search, signals from different targetdefining features can be combined to control the deployment of spatial attention during visual search for conjunctively defined targets when these features come from different dimensions (e.g., during search for red squares among blue squares and red circles). In contrast, the model assumes that no such guidance of attention by multiple target features is available when targets are defined by a combination of features from the same dimension (e.g., two different colours). According to the most recent version of Guided Search (Wolfe, 2007), only a single input signal from a particular feature dimension on the saliency map can be selectively weighted, and only one feature per dimension can therefore contribute to attentional guidance processes during visual search. This specific assumption is based on experiments demonstrating that search for targets defined by a conjunction of two colours or two orientations is much less efficient than search for colour/ shape conjunctions (Wolfe et al., 1990; see also Wing \& Allport, 1972). However, other studies have suggested that colour/colour conjunction search can be relatively efficient (Carrasco, Ponte, Rechea, \& Sampedro, 1998) and that pairs of colour/colour conjunction targets can be processed in parallel (Linnell \& Hymphreys, 2001). For example, Carrasco et al. (1998) showed that when all distractor objects share the same nontarget colour, practice led to highly efficient colour/colour conjunction search, presumably due to the colour-based perceptual grouping of distractors and the subsequent rapid rejection of these grouped non-target objects (see Linnell \& Hymphreys, 2001; 2002, for further evidence of within-dimension grouping effects in this type of search task). The assumption that colour/colour conjunction search cannot be guided by both target-defining features also has been challenged by behavioural and electrophysiological results from spatial cueing experiments (Irons, Folk, \& Remington, 2012; Grubert \& Eimer, in press), which suggest that search templates for multiple colours can be activated simultaneously. Thus, the question whether qualitatively different attentional guidance processes are activated when target-defining features come from the same or from different dimensions remains unresolved.

To obtain more precise insights into the cognitive and neural processes that are involved in the attentional selection of conjunctively defined targets during visual search and into the time course of these processes, event-related brain potential (ERP) measures can be employed. In particular, the N2pc component has been used in many recent studies as an electrophysiological marker for the attentional selection of objects with target-matching features in visual search tasks. The N2pc is an enhanced negativity over occipito-temporal electrode sites that emerge approximately $200 \mathrm{~ms}$ after stimulus onset contralateral to the side of space where a candidate target object is present among distractors in visual search displays and reflects the deployment of spatial selection of such objects (Eimer, 1996; Luck \& Hillyard, 1994). N2pc studies of visual search have typically used displays where only a single object with target-matching features was present. To use the N2pc as a marker of attentional selection processes that take place during search for conjunctively defined targets, this component needs to be measured in response to displays that contain the target as well as nontarget objects with some but not all targetdefining features. This requires search displays where some objects appear on the vertical meridian (i.e., above and below fixation). Because the N2pc is always elicited contralaterally to objects with target-matching features in the left or right visual field, no N2pc is triggered when such objects appear on the vertical meridian (Woodman \& Luck, 2003; Hickey, McDonald, \& Theeuwes, 2006; Eimer, Kiss, \& Nicholas, 2011; Eimer \& Grubert, 2014b). When a candidate target object is presented on the vertical midline together with another potential target on the horizontal midline, the N2pc will exclusively reflect the selection of the horizontal object, independent of the simultaneous attentional processing of the vertical object.

Because the N2pc component is time-locked to the onset of a search display, it is most suitable for investigating the time course of the attentional selection processes that are elicited 
rapidly after display onset. For this reason, most N2pc studies of visual search have used search displays where the detection and selection of possible target objects is relatively straightforward (e.g., displays where such candidate targets are feature singletons or otherwise perceptually salient, or displays with small set sizes). Even N2pc studies that investigated the time course of multiple target selection processes (Woodman \& Luck, 1999; Eimer \& Grubert, 2014b; Grubert \& Eimer, 2016) employed perceptually salient target objects. With larger set sizes or with displays where targets are more difficult to discriminate from distractors, the allocation of attention to candidate target objects is typically slower and more variable in time, which results in small and temporally smeared N2pc components.

In a recent study from our lab (Eimer \& Grubert, 2014a), the N2pc was used to investigate how attention is deployed during visual search for a conjunction of features from two different dimensions (colour and shape). Participants searched for targets that were defined by a specific colour/shape combination (e.g., blue squares) in search displays that contained two objects on the left and right side, and two objects on the vertical meridian (above and below fixation). On nocompetition trials, only one object with target-matching features was present, and this could either be the target or a distractor object that matched the target colour (e.g., a blue circle) or its shape (e.g., a red square). On competition trials, the target and a partially matching distractor object were both present in the same display, and one of these objects always appeared on a horizontal midline and the other on a vertical midline. This made it possible to measure the N2pc as a marker of the attentional selection of targets or of partially matching distractor objects on these competition trials. The early phase of the N2pc elicited by target objects was identical in size and onset latency to the sum of the two N2pc components triggered by colour-matching and shape-matching distractors. This additivity of the N2pc suggests that attention was initially allocated independently to both target-defining features. The observation that partially target-matching objects elicited additive N2pc components, also on competition trials when they were accompanied by a target in the same display, indicates that this initial feature-based deployment of attention operates in parallel and independently for different objects with targetmatching features. However, between 225-250 ms after search display onset, the N2pc to targets became larger than the summed contribution of the two N2pc components to colour-matching and shape-matching distractors. This emergence of a superadditive target N2pc was interpreted as marking the point in time where information started to be integrated across colour and shape, and attentional control processes became object-based (see Eimer \& Grubert, 2014a, for a more detailed discussion).

These N2pc results suggest that the attentional selection of targets defined by a conjunction of features from two different dimensions (colour and shape) is initially controlled in a parallel feature-based fashion before it becomes sensitive to the presence of feature conjunctions during a subsequent objectbased phase of attentional selectivity. The parallel nature of the initial feature-based phase of attentional object selection is not in line with the architecture of attentional mechanisms proposed by the Guided Search model. Although this model postulates that conjunction search is guided by a combination of signals from different feature channels, the attentional selection of individual objects is assumed to operate in a serial fashion (Wolfe, 2007). In the current study, we employed the $\mathrm{N} 2 \mathrm{pc}$ component to investigate the allocation of attention during visual search for targets that are defined by a combination of features from the same dimension (colour) to find out whether these processes are qualitatively similar or different from the attentional selection mechanisms that operate when targets are specified across feature dimensions. The procedures used in Experiment 1 were similar to those previously employed to investigate colour/shape conjunction search (Eimer \& Grubert, 2014a). Each search display contained four circular objects with different colours in their top and bottom halves (Fig. 1). Participants had to detect the presence or absence of a target object that was defined by a specific colour/ colour combination (e.g., red above green). The small display set size reduces the possibility that target selection was primarily based on the colour-based perceptual grouping of distractors, as previously found for colour/colour conjunction search with dense displays (Carrasco et al., 1998; Linnell \& Hymphreys, 2001). On no-competition trials, one targetmatching object (either the target or a distractor object that matched the top or bottom colour of the target, e.g., red above blue) was presented among three nonmatching, nontarget objects. On competition trials, the target and a partially matching distractor object were present in the same display, and one of these objects appeared on the horizontal midline and the other on the vertical midline. N2pc components were measured for targets and partially matching distractor objects, separately for no-competition and competition trials.

If attentional selection processes that are activated during colour/colour conjunction search are similar to the processes that operate when targets are defined across different feature dimensions, the pattern of N2pc results in Experiment 1 should be similar to that observed in the previous colour/ shape conjunction search experiment (Eimer \& Grubert, 2014a). The two types of distractors with a target-matching colour in their top or bottom halves should both elicit N2pc components, and the sum of these two N2pcs should initially equal the size of the N2pc elicited by targets. This would show that the early rapid deployment of attention was controlled in a feature-based fashion by independent guidance signals associated with two different target-defining attributes within the same dimension. Furthermore, partially target-matching distractors should elicit reliable and additive N2pc 


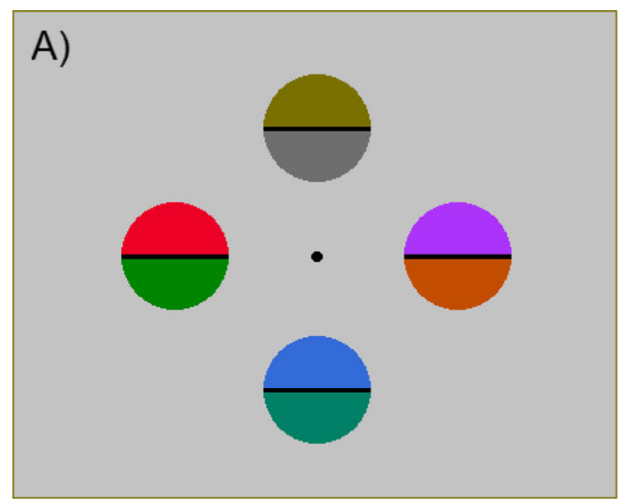

Fig. 1 Example illustration of search displays on no-competition (a) and competition (b) trials in Experiment 1. On no-competition trials, either a target or partially matching distractor object appeared among three non-target items, positioned on the vertical or horizontal midline. On competition trials, both a target and partially matching distractor object were present on each trial,

components also on competition trials, despite the fact that a target object was simultaneously present on these trials, indicating that attention was allocated independently and in parallel to all objects with target-matching colours. During a later phase of attentional processing, the target N2pc may become larger than the sum of the two N2pcs to partially matching distractors, with the emergence of such a superadditive N2pc marking the transition from independent feature-based to integrated object-based attentional control processes (Eimer \& Grubert, 2014a). An alternative possibility is that in contrast to search for targets defined across different dimensions, attentional selection mechanisms cannot be guided jointly by features from the same dimension, as proposed by the Guided Search model (Wolfe, 2007). In this case, a different pattern of N2pc results should be observed in Experiment 1. For example, if targets and partially target-matching distractor objects did not differ in their ability to attract attention, N2pc components should be similar in size for these two types of objects throughout the N2pc measurement window.

\section{Experiment 1}

\section{Method}

\section{Participants}

Fifteen paid participants were recruited for Experiment 1 . Three participants were excluded due to excessive artifacts during EEG recording (two for eye blinks on more than $50 \%$ of all trials, one for horizontal eye movements on more than $35 \%$ of all trials), leaving a final sample of 12 participants (mean age $=31$ years, $\mathrm{SD}=6$; six males; two lefthanded). All participants had normal or corrected-to-normal vision, reported normal colour vision, and were naïve to the experimental hypotheses.

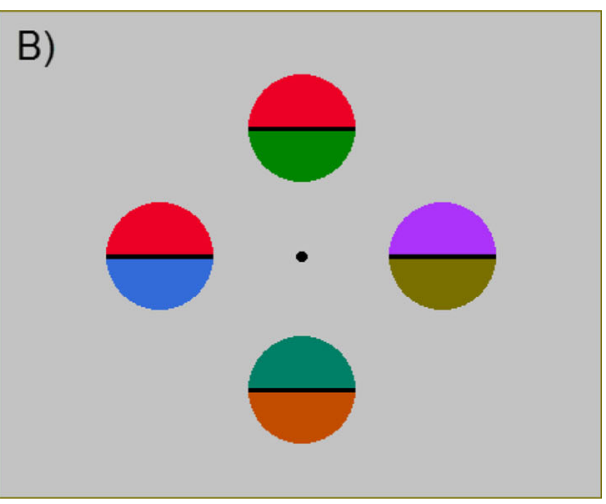

always appearing on opposite midline axes. Participants responded to the presence or absence of the target object (in this example, the red-abovegreen object). Partially matching distractor objects (e.g., the red-above-blue distractor in B) matched the colour of the target in either its top or bottom half. See the online article for the colour version of this figure

\section{Stimuli and procedure}

E-Prime (Psychology Software Tools, Inc.) was used to create and execute the experiment, presented on a 24-inch Samsung LCD monitor $(100 \mathrm{~Hz} ; 1280 \times 1024$ screen resolution) at a viewing distance of about $90 \mathrm{~cm}$, and controlled on an LG computer PC. Participants' manual responses were recorded via keyboard button presses. Each trial in the experiment consisted of stimuli presented on a light grey background, with a black fixation dot $\left(0.2^{\circ} \times 0.2^{\circ}\right.$ of visual angle $)$ appearing constantly throughout each block. In all search displays, four coloured circular objects were presented above, below, to the left, and to the right of fixation at an eccentricity of $1.2^{\circ}$. Each object measured $1.2^{\circ} \times 1.2^{\circ}$, and contained two equally large top and bottom portions that were presented in two different colours. The colours used in the experiment were red (CIE x/y coordinates: $0.621 / 0.333)$, orange $(0.550 / 0.393)$, gold $(0.401 / 0.482)$, green $(0.270 / 0.562)$, cyan $(0.217 / 0.358)$, blue $(0.183 / 0.183)$, magenta $(0.272 / 0.164)$, and dark grey (0.288/0.312). All colours were equiluminant $\left(30 \mathrm{~cd} / \mathrm{m}^{2}\right)$, measured using a Konica Minolta CS-100A luminance and colour meter. The centre of each colour object contained a small horizontal black line separating the two colours (Fig. 1).

Each search array was presented for 100 ms. Participants' task was to respond to the presence or absence of a predefined colour object combination (e.g., red above green circle) using the numeric keypad (" 1 " for present, " 2 " for absent) as quickly and as accurately as possible. The two target-defining colours were selected randomly and independently for each participant, with the exception that dark grey never served as one of the target colours. The different types of search displays are illustrated in Fig. 1. Targets were present on $50 \%$ of all trials. There were two types of task-irrelevant objects. Nontarget objects contained two task-irrelevant colours. Partially matching distractor objects contained one of the two targetdefining colours in its correct position within the object, but 
together with another task-irrelevant colour (e.g., red above blue when the target was red above green). On no-competition trials, search displays contained a single object with targetmatching features, which could either be the target itself or a partially matching distractor. The target or partially matching distractor appeared with equal probability and unpredictably on the horizontal or vertical midline on no-competition trials. On competition trials, a target and partially matching distractor both appeared in the same display together with two other nontarget objects. On half of these trials, the target appeared on the horizontal midline and the partially matching distractor on the vertical midline, and these positions were reversed for the other half of all competition trials. With the exception of competition trials where a distractor object shared one colour with the target, two identical colours never appeared within the same display. No-competition and competition trials were presented in random order in all blocks. Participants had to execute a response within a 2,000-ms interval. Once a response was registered, there was an intertrial interval of 1,500 $\mathrm{ms}$ before the search display on the next trial was presented.

Participants completed a practice block of 12 trials, before undertaking 16 experimental blocks of 96 trials. Within each block, there were 48 target-present and 48 target-absent trials. On target-present trials, targets appeared equally often on the vertical midline (24) or the horizontal midline (24). For 32 of 48 target-present trials, a partially matching distractor also was present in the opposite axis to the target. On target-absent trials, 32 of 48 trials contained a partially matching distractor object, and the remaining 16 trials contained four nontarget objects with two task-irrelevant colours.

\section{EEG recording and data analysis}

EEG was DC-recorded from 27 scalp electrodes, mounted on an elastic cap at sites Fpz, F7, F8, F3, F4, Fz, FC5, FC6, T7, T8, C3, C4, Cz, CP5, CP6, P9, P10, P7, P8, P3, P4, Pz, PO7, PO8, PO9, PO10, and Oz. A 500-Hz sampling rate with a 40$\mathrm{Hz}$ low-pass filter was used, with no other offline filters applied. Channels were referenced online to a left-earlobe electrode, and re-referenced offline to an average of both earlobes. Trials with eye-movement (exceeding $\pm 30 \mu \mathrm{V}$ in the HEOG channels), eye blink (exceeding $\pm 60 \mu \mathrm{V}$ at Fpz) and muscle movement artifacts (exceeding $\pm 80 \mu \mathrm{V}$ at all other channels) were rejected. This resulted in an average data loss of $10 \%$ of all trials $(\mathrm{SD}=7)$. The remaining trials were segmented into epochs from $-100 \mathrm{~ms}$ before and $500 \mathrm{~ms}$ after search array onset. For each type of search display that contained a target or partially matching distractor item presented on the horizontal midline, averaged ERP waveforms were computed. N2pc amplitudes were quantified based on ERP mean amplitudes obtained between 190 and $290 \mathrm{~ms}$ after search array onset at posterior electrode sites PO7 and PO8. N2pc onset latencies were determined by assessing difference waveforms (contralateral minus ipsilateral ERPs) using a jackknife-based analysis method (Miller, Patterson \& Ulrich, 1998). Onset latencies were defined for these difference waves using a relative onset criterion of $50 \%$ of $\mathrm{N} 2 \mathrm{pc}$ peak amplitudes, and onset latencies were compared using paired $t$ tests with $t$ values corrected according to the formula described by Miller et al. (1998).

\section{Results}

\section{Behavioural data}

Reaction times on trials with partially matching distractor objects did not differ as a function of whether the matching colour appeared in the upper or lower portion, for either target-present trials $(M=527 \mathrm{~ms}$ vs. $527 \mathrm{~ms}, t(11)<1)$, or target-absent trials $(M=510 \mathrm{~ms}$ vs. $504 \mathrm{~ms}, t(11)<1)$. Therefore, the RT data were averaged across these trials. Data were entered into a $2 \times 2$ repeated-measures Analysis of Variance (ANOVA) with the factors Target (present, absent) and Partially Matching Distractor (present, absent). Analysis showed a main effect of Target $\left(F(1,11)=29.53, p<0.001, \eta_{\mathrm{p}}{ }^{2}=0.73\right)$, with generally faster reaction times on target-absent trials $(M=489 \mathrm{~ms}$, $M S E=13$ vs. $M=522 \mathrm{~ms}, M S E=12)$. In addition, a main effect of Partially Matching Distractor $(F(1,11)=64.49$, $\left.p<0.001, \eta_{\mathrm{p}}{ }^{2}=0.85\right)$ showed that reaction times were generally slowed on trials where a partially matching item was present $(M=517 \mathrm{~ms}, M S E=13$ vs. $M=495 \mathrm{~ms}, M S E=11)$ A significant interaction between these two factors $(F(1,11)=$ 117.88, $p<0.001, \eta_{\mathrm{p}}{ }^{2}=0.92$ ) also was found, demonstrating that the RT costs produced by the presence of a partially matching distractor was more pronounced on target-absent $(M$ diff $=35 \mathrm{~ms} ; t(11)=12.57, p<0.001)$ than on targetpresent trials $(M$ diff $=10 \mathrm{~ms} ; t(11)=2.90, p<0.02)$.

Error rates were entered into a matching ANOVA model. As for reaction times, there was no difference in error rates for partially matching distractors with a target-matching colour in the upper or lower part on target-present $(M=2 \%$ vs. $3 \%$; $t(11)<1)$ or target-absent trials $(M=4 \%$ vs. $4 \% ; t(11)<1)$, and so these data were collapsed. Analysis showed a main effect of Target $\left(F(1,11)=11.40, p<0.01, \eta_{\mathrm{p}}{ }^{2}=0.51\right)$, with more missed targets on target-present trials than False Alarms on target-absent trials $(M=4 \%$ vs. $1 \%)$. A main effect of Partially Matching Distractor $(F(1,11)=24.50, p<0.001$, $\left.\eta_{\mathrm{p}}{ }^{2}=0.69\right)$ showed that response errors were generally more frequent when a partially matching item was present $(M=3 \%$ vs. $2 \%$ ). Finally, a significant interaction between both factors $\left(F(1,11)=6.53, p<0.03, \eta_{\mathrm{p}}{ }^{2}=0.37\right)$ was due to the fact that the presence of partially matching items increased False Alarm rates on target-absent trials $(M$ diff $=2 \% ; t(11)=$ $4.31, p=0.001$ ), but had no significant effect on the percentage of missed targets on target-present trials ( $M$ diff $=1 \%$; $t(11)=1.68, p>0.10)$. 


\section{N2pc components on no-competition trials}

Figure 2 (top panel) shows ERPs triggered in response to search displays that contained a target object or a partially matching distractor (shown separately for trials where the target-matching colour was present in the top or bottom half of these objects). Clear N2pc components were elicited by targets. Partially matching distractors also triggered N2pc components, but they were reduced in size relative to the target N2pc. To assess the size of N2pc components on nocompetition trials, mean ERP amplitudes obtained in the 190 to $290 \mathrm{~ms}$ poststimulus time window were entered into a $2 \mathrm{x} 2$ repeated-measures ANOVA with the factors Item (horizontal target and horizontal partially matching distractor, averaged across partially matching items with a target-matching colour at the top or bottom) and Laterality (ipsilateral, contralateral). There was no main effect of Item $(F(1,11)=1.04, p>0.30$, $\left.\eta_{\mathrm{p}}{ }^{2}=0.09\right)$. However, there was a significant main effect of Laterality $\left(F(1,11)=37.90, p<0.001, \eta_{\mathrm{p}}{ }^{2}=0.78\right)$, reflecting the presence of reliable N2pc components (Fig. 2). Critically, a significant Item $\mathrm{x}$ Laterality interaction $(F(1,11)=46.38, p<$ $\left.0.001, \eta_{\mathrm{p}}{ }^{2}=0.81\right)$ demonstrated that $\mathrm{N} 2 \mathrm{pc}$ components were larger for target stimuli than for partially matching distractors. However, paired-sample $t$ tests showed that reliable N2pc components were in fact elicited to both targets $(M \operatorname{diff}=$ $-2.07 \mu \mathrm{V} ; t(11)=6.69, p<0.001)$ and partially matching distractors $(M$ diff $=-0.64 \mu \mathrm{V} ; t(11)=4.20, p=0.001)$.

To assess whether, and up to what point, the target N2pc equalled the sum of the N2pc components to the two types of partially matching distractor objects, N2pc difference waveforms (i.e., contralateral minus ipsilateral ERP waveforms) computed for distractors with a target-matching colour at the top or bottom were summed and compared to the N2pc difference waveforms obtained for target objects (Fig. 2, bottom panel). A jackknife-based onset latency comparison with a relative onset criterion (50\% of N2pc peak amplitudes) showed no significant difference between these N2pc waveforms (220 ms versus $214 \mathrm{~ms}$ poststimulus for targets versus partially matching distractors; $\left.t_{c}(11)<1\right)$. As shown in Fig. 2 (bottom panel), the summed N2pc components to the two types of partially matching distractors was initially equal in size to the target N2pc, before the N2pc to the target started to become superadditive (i.e., larger than the sum of the two $\mathrm{N} 2$ pc components to partially matching distractors). To determine the point in time when the target N2pc became superadditive, a double-difference N2pc waveform was created by subtracting the summed distractor N2pc from the N2pc elicited by target stimuli. A jackknife-based onset analysis using the same $50 \%$ peak amplitude criterion showed that the target N2pc started to become larger than the summed partially matching distractor N2pc at a poststimulus latency of $262 \mathrm{~ms}$. To obtain further evidence for this transition from additive to superadditive target N2pc components, N2pc mean amplitudes (based on N2pc difference waveforms) to targets and summed partially matching distractors were obtained within two poststimulus time windows (190-240 ms and 240-290 ms). A 2x2 ANOVA with the factors Item (target, summed partially matching distractor) and Time Window (early, late) demonstrated a significant Item $\mathrm{x}$ Time Window interaction $\left(F(1,11)=44.45, p<0.001, \eta_{\mathrm{p}}{ }^{2}=0.80\right)$, in line with the observation that a superadditive target $\mathrm{N} 2 \mathrm{pc}$ emerged during the late phase of the N2pc component.
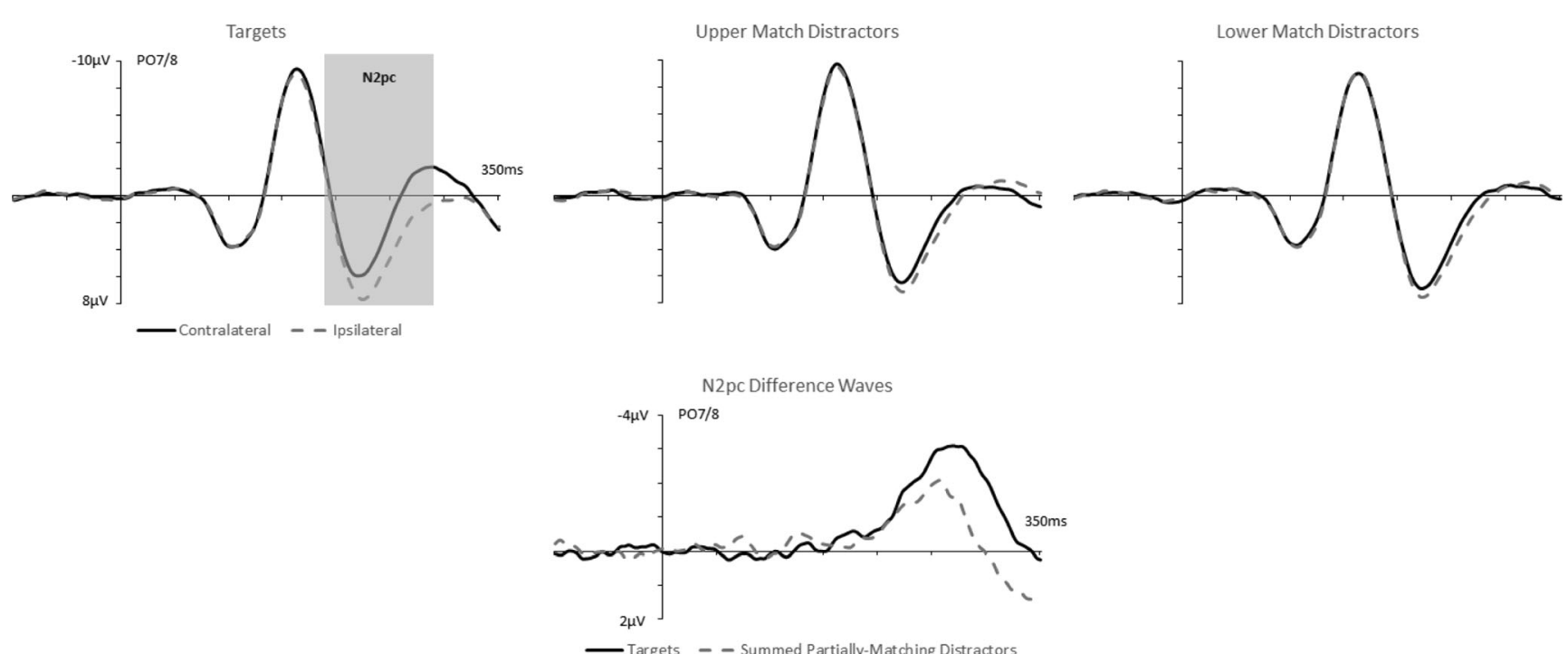

Fig. 2 Top panel: Grand-average event-related brain potentials (ERPs) obtained on no-competition trials in Experiment 1, in the 350-ms interval poststimulus onset at electrode sites PO7/8 contralateral and ipsilateral to the location of lateral target objects or lateral distractor objects with a target- matching colour in their upper or lower half. Bottom panel: N2pc difference waveforms (ipsilateral minus contralateral ERPs) for target items (solid line), and summed N2pc difference waves for distractor items with a targetmatching colour at the upper or lower position (dashed line) 


\section{N2pc components on competition trials}

Figure 3 (top panel) shows ERPs triggered in response to search displays that contained a horizontal target object and a partially matching distractor on the vertical midline, and displays with a horizontal partially matching distractor (shown separately for objects with the targetmatching colour in the top or bottom half) and a vertical target. Targets triggered clear N2pc components, and partially matching distractors also appeared to elicit an N2pc, which was again reduced in size relative to the target $\mathrm{N} 2$ pc. A 2x2 repeated-measures ANOVA for N2pc mean amplitudes showed no main effect of Item $(F(1,11)=$ $1.92, p>0.10)$ but a significant effect of Laterality $\left(F(1,11)=34.07, p<0.001, \eta_{\mathrm{p}}{ }^{2}=0.76\right)$. The presence of an Item x Laterality interaction $(F(1,11)=51.36, p<$ $0.001, \eta_{\mathrm{p}}{ }^{2}=0.82$ ) showed that targets elicited a larger $\mathrm{N} 2$ pc than partially matching distractors. However, reliable N2pc components were triggered not only by targets $(M$ diff $=-1.82 \mu \mathrm{V} ; t(11)=6.55, p<0.001)$, but, importantly, also by partially matching distractors $(M$ diff $=$ $-0.40 \mu \mathrm{V} ; t(11)=3.31, p<0.01)$.

As shown in the N2pc difference waveforms for competition trials in Fig. 3 (bottom panel), the summed $\mathrm{N} 2 \mathrm{pc}$ component to the two types of partially matching distractors was initially similar in size to the target N2pc, before the N2pc to the target started to become superadditive. $\mathrm{N} 2 \mathrm{pc}$ onset latencies for targets and summed partially matching distractors were compared using a jackknife-based procedure with a relative onset criterion ( $50 \%$ of peak amplitude). Onset latencies were identical $(221 \mathrm{~ms})$ for the target $\mathrm{N} 2 \mathrm{pc}$ and the summed N2pc to partially matching distractors $\left(t_{c}(11)<1\right)$. To determine the point in time when the target $\mathrm{N} 2 \mathrm{pc}$ became superadditive on competition trials, a doubledifference N2pc waveform was created by subtracting the summed N2pc to both types of partially matching distractors from the target N2pc. A jackknife-based onset analysis using the same relative onset criterion showed that the target N2pc started to become larger than the summed partially matching distractor N2pc at a post-stimulus latency of $252 \mathrm{~ms}$. To confirm this observation, N2pc mean amplitudes (based on N2pc difference waveforms) to targets and summed partially matching distractors were obtained and compared within two successive post-stimulus time windows (early N2pc: 190-240 ms; late N2pc: 240-290 ms post-stimulus). Similar to no-competition trials, this analysis revealed a significant Time Window $\mathrm{x}$ Item interaction $(F(1,11)=15.68, p<0.01$, $\left.\eta_{\mathrm{p}}{ }^{2}=0.59\right)$, reflecting the observation that $\mathrm{N} 2 \mathrm{pc}$ amplitude differences between targets and summed partially matching distractors emerged during the later phase of the N2pc component on competition trials.

\section{Discussion of Experiment 1}

The pattern of $\mathrm{N} 2 \mathrm{pc}$ results obtained in Experiment 1 during colour/colour conjunction search was very similar to the results previously observed in a colour/shape conjunction search task (Eimer \& Grubert, 2014a), suggesting that attentional selection mechanisms operate in a qualitatively similar fashion when target objects are defined by features from the same or two different dimensions. Reliable N2pc components were elicited not only by target objects but also by distractors that matched one but not both target-defining colours, demonstrating that these partially matching items were able to attract
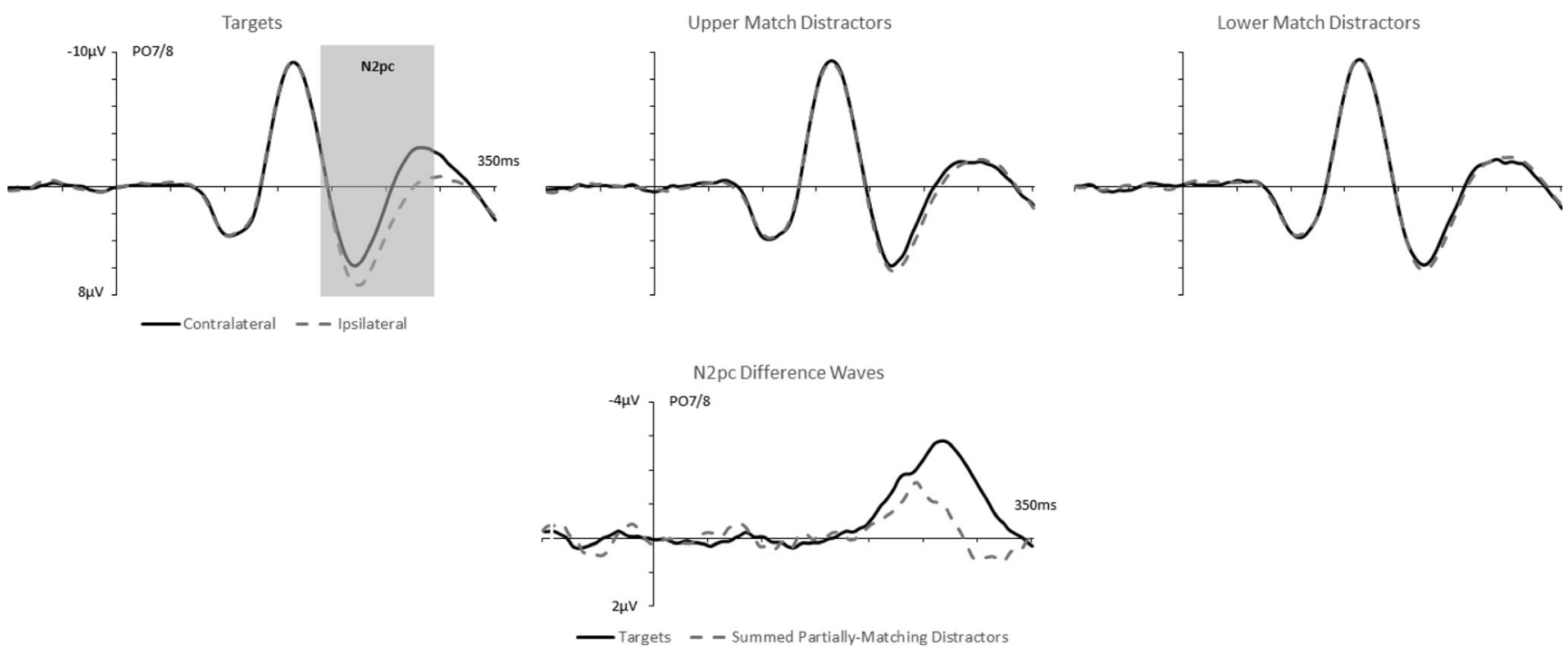

Fig. 3 Top panel: Grand-average event-related brain potentials (ERPs) obtained on competition trials in Experiment 1, in the 350-ms interval poststimulus onset at electrode sites $\mathrm{PO} / / 8$ contralateral and ipsilateral to the location of lateral targets or distractors with an upper or lower target- matching colour. Bottom panel: N2pc difference waveforms (ipsilateral minus contralateral ERPs) for target (solid line), and summed N2pc difference waves for distractor items with a target-matching colour at the upper or lower position (dashed line) 
attention. Importantly, N2pc components to distractor objects with a target-matching colour at the top or bottom emerged at the same time as the target $\mathrm{N} 2 \mathrm{pc}$, and the sum of these two $\mathrm{N} 2 p c s$ to partially matching distractors was initially similar in size to the N2pc to target objects. At approximately $250 \mathrm{~ms}$ after search display onset, the target $\mathrm{N} 2 \mathrm{pc}$ became larger than the sum of the N2pc components to partially matching distractors. This was the case not only on no-competition trials where only a single object with target-matching features was present in the search display but also on competition trials where a target and a partially matching distractor appeared simultaneously. This temporal pattern of an initially additive target $\mathrm{N} 2 \mathrm{pc}$ that then became superadditive matches the results from a previous study where targets were defined by a combination of colour and shape (Eimer \& Grubert, 2014a). These N2pc results suggest that attention was at first allocated independently to individual targetmatching feature, irrespective of whether the other target feature also was present in the same object.

The fact that N2pc components to partially matching distractor objects that were accompanied by a target in the same display emerged at the same time as the target $\mathrm{N} 2 \mathrm{pc}$, and the observation that the sum of these components was initially equal in size to the target $\mathrm{N} 2 \mathrm{pc}$, both suggest that the rapid allocation of attention to target-matching colours during colour/colour conjunction search can operate simultaneously for multiple target colours in the visual field. This finding is problematic for the assumption that attention can be guided by only one feature per dimension (Wolfe, 2007) but is consistent with the alternative hypothesis that attention can be set for multiple colours simultaneously (Irons et al., 2012). Furthermore, the presence of reliable N2pc components to partially matching distractors in competition trials strongly suggests that attention was deployed concurrently to different objects with target-matching colours in the same search display. This is not in line with the hypothesis that objects are selected in a strictly serial fashion for entry into a subsequent object recognition system and that attention is first allocated to the object that produces the strongest activation on the saliency map (Wolfe, 2007). If this was the case, partially matching distractors should not have attracted attention and therefore not have triggered N2pc components under conditions where a stronger competitor for attentional selection (the vertical target) was present in the same display. If the allocation of attention to a partially matching distractor was entirely independent of a second parallel attentional deployment to the target object in the same display, N2pc amplitudes to these distractors should not differ between competition trials and no-competition trials where no such additional attentional selection process was activated. In fact, N2pc components to partially matching distractors were numerically larger on no-competition trials ( $M$ diff $=-1.27$ vs. $-0.81 \mu \mathrm{V})$. Although this difference only approached statistical significance $(t(11)=2.14, p=0.055)$, it indicates that the attentional selection of targets and partially matching nontargets did not operate entirely independently of each other. The trend towards an attenuation of $\mathrm{N} 2 \mathrm{pc}$ amplitudes on competition trials suggests the existence of competitive interactions between two simultaneously active foci of spatial attention, which reduces the spatially selective bias on visual processing at each attended location.

At approximately $250 \mathrm{~ms}$ after search display onset, the target N2pc became superadditive (Figs. 2 and 3), demonstrating that during this later phase of attentional object selection, the spatially selective activation elicited by target objects with both taskrelevant colours became stronger than the sum of the activations triggered by objects with only one target-matching colour. Analogous to similar observations for colour/shape conjunction search (Eimer \& Grubert, 2014a), this shows that at some point during the spatially selective attentional processing of these objects, attentional control processes can detect the presence of multiple task-relevant features at a particular object location, and assign greater priority to objects that possess all of these features. The emergence of a superadditive target N2pc suggests that an initial parallel feature-based phase of attentional selectivity is followed by a second phase where the deployment of attention starts to be controlled by the integration of different target features within the same object. If attentional guidance was limited to a single feature within a given dimension (Wolfe, 2007), no such superadditivity of the N2pc to target objects should have been observed, as the integration of attentional processing across two different target-defining colours requires that both features are simultaneously available to attentional control processes.

The exact nature of the processes that are responsible for the emergence of a superadditive target N2pc during visual search for colour/shape conjunctions (Eimer \& Grubert, 2014a) and for colour/colour conjunctions (the current Experiment 1) needs further clarification. This effect could reflect the spatially selective facilitation of target processing but also could be linked to a reduction of the spatial bias triggered by distractors over time. A spatially selective facilitation of target processing could be the result of combining the target-matching features of the same object as an integrated object representation at an object-based stage of attentional selectivity that follows the initial parallel feature-based stage. Alternatively, the emergence of N2pc amplitude differences between targets and summed partially matching distractors at approximately $250 \mathrm{~ms}$ after search display onset may reflect a reduction in the size of the $\mathrm{N} 2 \mathrm{pc}$ components triggered by these distractors at this moment in time. For example, attention may start to be disengaged from objects that possess only one target-matching feature once the status of these objects as task-irrelevant distractors has been registered (see Kiss, Grubert, \& Eimer, 2013, and Berggren \& Eimer, in press, for evidence of such attentional disengagement processes from spatial cueing experiments). Participants may have had a strong incentive to rapidly 
disengage attention from partially matching distractors, because these objects were frequently accompanied by a fully matching target in the same display. If this was the case, the superadditive target N2pc observed in Experiment 1 would be the result of the target $\mathrm{N} 2 \mathrm{pc}$ component persisting and the initial N2pc to partially matching distractor items declining as attention is withdrawn from their location.

Experiment 2 was conducted to test these alternative explanations for the superadditivity of the target N2pc. Procedures were similar to Experiment 1, except that objects with one or two target-matching colours were now both task-relevant. Participants searched for target objects defined by two possible colours (e.g., red and green). On different trials, search displays contained an object that matched one of these two colours (single-feature targets; e.g., a red and blue object), or both colours (dual-feature targets). Both types of objects were designated as targets in Experiment 2. Participants' task was to report whether the target object on any given trial matched one or both targetdefining colours. These targets were accompanied by three objects with two task-irrelevant colours on each trial. There were no nontarget trials and no trials where single-feature and dualfeature targets appeared together in the same display (as was the case for the competition trials of Experiment 1). N2pc components were measured separately for single-feature and dualfeature targets. If the early phase of attentional selectivity during visual search for colour conjunctions always operates independently for individual colours, the N2pc to dual-feature targets should initially be equal in size to the sum of the N2pc components to the two single-feature targets, analogous to Experiment 1. The critical new question was whether a superadditive N2pc to dual-feature targets would still emerge in Experiment 2, in spite of the fact that objects with one target colour also were now taskrelevant and required a response. If the superadditivity observed in Experiment 1 reflected the withdrawal of attention from taskirrelevant partially target-matching distractor objects, no such withdrawal should be found for single-feature target objects in Experiment 2. In this case, the summed N2pc components to single-feature targets should remain equal in size (or perhaps even become larger) than the N2pc to dual-feature targets. On the other hand, the presence of a superadditive N2pc to dualfeature targets in Experiment 2 would rule out this attentional withdrawal account, and would support an alternative interpretation in terms of object integration.

\section{Experiment 2}

\section{Method}

\section{Participants}

Thirteen paid participants took part in Experiment 2. One participant was excluded due to excessive alpha activity and eye blinks (removing more than $50 \%$ of all trials), leaving a final sample of 12 participants (mean age $=29$ years, $\mathrm{SD}=6 ; 5$ males; 2 left-handed). All participants had normal or corrected-to-normal vision, reported normal colour vision, and were naïve to the experimental hypotheses.

\section{Stimuli and procedure}

Experiment 2 matched the setup of Experiment 1 with the following exceptions. The experiment was presented on a 24-inch BenQ monitor $(60 \mathrm{~Hz} ; 1920$ x 1080 screen resolution) at a viewing distance of approximately $90 \mathrm{~cm}$, and controlled on a SilverStone computer PC. The colours used in the experiment were red (CIE $\mathrm{x} / \mathrm{y}$ coordinates: $0.605 / 0.322)$, orange $(0.543 / 0.409)$, gold $(0.405 / 0.470)$, green $(0.296 / 0.604)$, cyan $(0.227 / 0.324)$, blue $(0.227 / 0.324)$, magenta $(0.270 / 0.134)$, and dark grey $(0.305 / 0.325)$. All colours were equiluminant $\left(14 \mathrm{~cd} / \mathrm{m}^{2}\right)$. As in Experiment 1, search displays contained four circular objects with two different colours in their top and bottom halves that were presented in the left, right, top, and bottom positions. There were again two predefined target colours for each participant, but task instructions were different. Participants had to report on each trial whether the target object in the search display matched one or both target colours, using the numeric keypad ("1" for single-feature match, "2" for dual-feature match). All search displays contained one target object among three task-irrelevant non-targets, with singlefeature and dual-feature targets presented in random order and with equal probability in each block. To maximize the number of trials available for measuring N2pc components, these two types of targets were always presented on the left or right side and never at the top or bottom positions. Participants completed two blocked versions of this task. In five successive blocks, the two target colours occupied a constant position within the respective target objects, as in Experiment 1 (e.g., red above green for dual-feature targets, and red above blue or magenta above green for single-feature targets). In the other five successive blocks, there were no fixed colourlocation mappings (e.g., red and green as target-defining colours were equally likely to appear in the top or bottom positions of single-feature or dual-feature targets). Following practice, participants completed 10 experimental blocks of 96 trials each ( 5 blocks with fixed colourlocation mappings followed by 5 blocks with varied mappings, or vice versa, counterbalanced across participants). The two target-defining colours were selected randomly for each participant and remained the same in both versions of the task for each individual participant. Within each block, dual-feature targets appeared on $50 \%$ 
of trials, and the two types of single-feature targets appeared on the remaining $50 \%$ of trials.

\section{EEG recording and data analysis}

EEG recording and analysis was identical to Experiment 1. The average rate of trials removed due to artefact rejection was $9 \%$ ( $\mathrm{SD}=11$ ). Averaged ERP waveforms were computed for displays with dual-feature targets and for the two types of single-feature target displays (labelled colour 1 and colour 2, with colour labels randomly assigned to each of the two target colours for each participant), separately for the two blocked spatial configuration conditions.

\section{Results}

\section{Behavioural data}

A two-way repeated-measures ANOVA conducted for RTs with the factors Target (single-feature, dual-feature) and Configuration (set, varied) showed no main effect of Configuration or an interaction between Configuration and Target (both F's $<1$ ). However, there was a main effect of Target $\left(\mathrm{F}(1,11)=33.51, p<0.001, \eta_{\mathrm{p}}{ }^{2}=0.75\right)$, as RTs were faster for dual-feature compared to single-feature targets $(\mathrm{M}=588$ vs. $633 \mathrm{~ms})$. A matching ANOVA performed on error rates showed no main effect of Target $(\mathrm{F}<1)$, Configuration $(\mathrm{F}(1,11)=3.13, p>0.10)$, and no interaction between these factors $(\mathrm{F}<1)$.

\section{N2pc components}

Figure 4 (top panel) shows ERPs triggered in response to search displays with dual-feature targets and displays that contain one of the two single-feature targets (colour 1 and colour 2, randomly assigned for each participant). N2pc components were elicited by both types of targets but were larger for dual-feature as compared to single-feature targets. An initial three-way repeated-measures ANOVA was conducted on mean ERP amplitudes obtained in the 190 to 290 ms poststimulus time window, with the factors Target (single-feature, dual-feature), Configuration (set, varied), and Laterality (ipsilateral, contralateral). As Configuration did not interact with Target $(\mathrm{F}<1)$, Laterality $(\mathrm{F}<1)$, or as part of a three-way interaction $(\mathrm{F}(1,11)=3.05, p>0.10)$, subsequent analyses were based on ERP data that were averaged across blocks with set and varied configurations of target colours. A new $2 \times 2$ ANOVA using the factors Target and Laterality showed no main effect of Target $(\mathrm{F}<1)$ but a significant Target $\mathrm{x}$ Laterality interaction $\left(\mathrm{F}(1,11)=55.04, p<0.001, \eta_{\mathrm{p}}{ }^{2}=0.83\right)$. Whereas both types of targets elicited a significant $\mathrm{N} 2 \mathrm{pc}$ component (all $t>4.62$, all $p \leq 0.001$ ), dual-feature targets $(\mathrm{M}$ diff $=-1.77 \mu \mathrm{V})$ elicited a larger $\mathrm{N} 2 \mathrm{pc}$ compared with single-feature targets $(\mathrm{M}$ diff $=-0.59 \mu \mathrm{V})$.
To examine whether and up to which point in time the N2pc to dual-feature targets equalled the sum of the two N2pc components to single-feature targets, the two N2pc waveforms to the two types of single-feature targets (i.e., colours 1 and 2) were summed and compared to the N2pc to dual-feature targets. The corresponding N2pc difference waveforms to dual-feature and summed single-feature targets shown in Fig. 4 (bottom panel) reveal a pattern that is very similar to Experiment 1 . Both N2pc waveforms started at the same time, but the later phase of the $\mathrm{N} 2 \mathrm{pc}$ to dual-feature targets was again superadditive (i.e., larger than the summed N2pcs to single-feature targets). A jackknifebased onset latency comparison with a relative onset criterion (50\% of N2pc peak amplitudes) showed no significant difference between these N2pc waveforms (216 ms vs. $212 \mathrm{~ms}$ poststimulus for dual-feature and summed single-feature targets; $\left.t_{c}(11)=1.13, p>0.25\right)$. To determine the point in time when the dual-feature target N2pc became superadditive, a doubledifference N2pc waveform was created by subtracting the summed single-feature target $\mathrm{N} 2 \mathrm{pc}$ from the dual-feature target $\mathrm{N} 2 \mathrm{pc}$. A jackknife-based onset analysis using the same relative onset criterion showed that the dual-feature target $\mathrm{N} 2 \mathrm{pc}$ started to become larger than the summed single-feature target N2pcs at a poststimulus latency of $249 \mathrm{~ms}$. The emergence of a superadditive $\mathrm{N} 2 \mathrm{pc}$ to dual-feature targets during the late phase of the N2pc was further confirmed by an analysis of N2pc mean amplitudes to dual-feature targets and summed single-feature targets that were obtained within two successive post-stimulus time windows (early N2pc: 190-240 ms; late N2pc: 240-290 ms poststimulus). As in Experiment 1, this analysis revealed a significant Time Window $\mathrm{x}$ Target interaction $(\mathrm{F}(1,11)=8.39$, $p<0.02, \eta_{\mathrm{p}}^{2}=0.43$ ).

\section{Discussion of Experiment 2}

Experiment 2 tested whether the emergence of a superadditive target N2pc observed in Experiment 1 was linked to the integration of attentional processing across the two task-relevant colours of target objects, or instead reflected the disengagement of attention from partially matching distractor objects once these objects were recognized as task-irrelevant. In Experiment 2, objects with one or two target colours were both task-relevant, and these two types of objects never appeared in the same display. If the superadditive target N2pc in Experiment 1 was due to a selective withdrawal of attention from distractor objects with one target-matching colour, no such superadditivity should have been observed in Experiment 2 for the N2pc to dual-feature targets as compared to summed single-feature targets. In fact, the temporal pattern of N2pc components in Experiment 2 was very similar to Experiment 1 . The dual-feature target N2pc emerged at the same time and was initially equal in size to the sum of the two N2pc components to single-feature targets and then became larger than the summed single-feature N2pc 


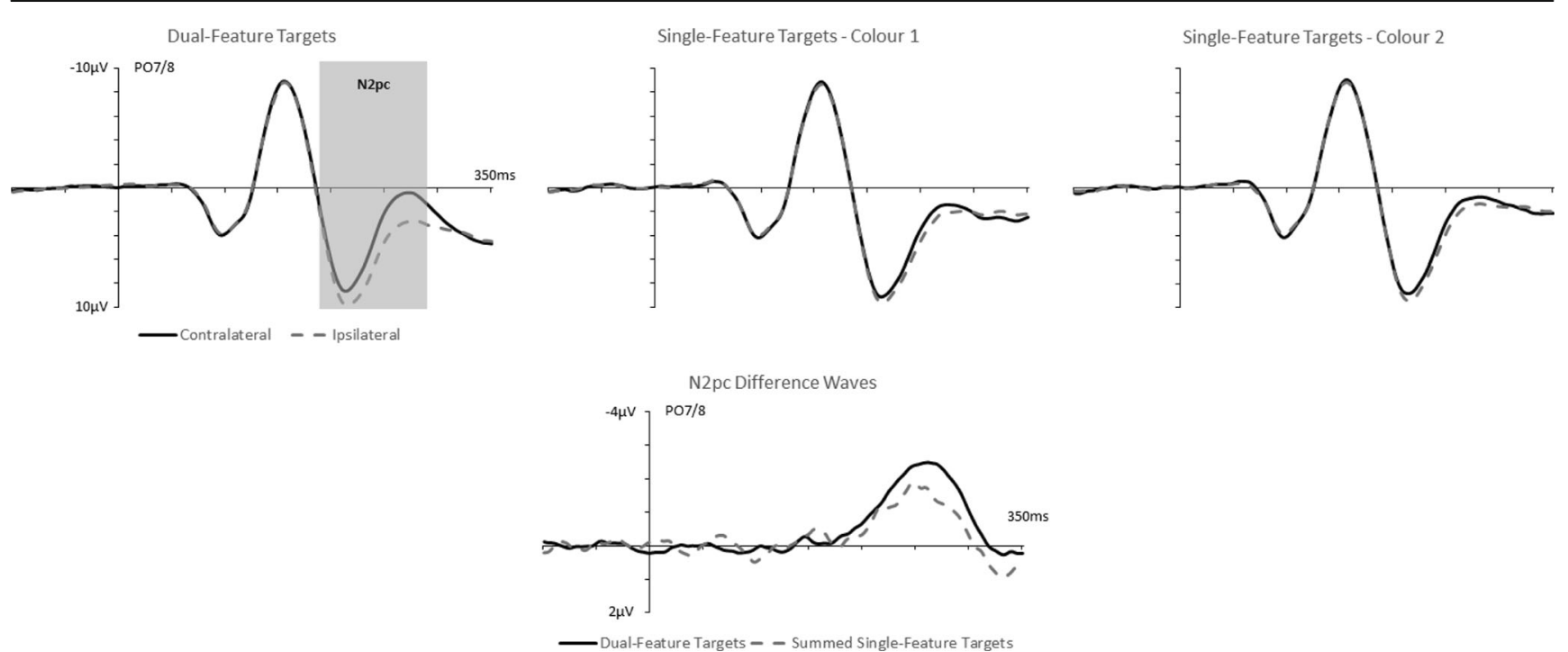

Fig. 4 Top panel: Grand-average event-related brain potentials (ERPs) in Experiment 2, obtained in the 350-ms interval poststimulus onset at electrode sites PO7/8 contralateral and ipsilateral to the location of dualfeature targets, or single-feature targets that had one of the two task- relevant colours (colour 1 or colour 2, selected randomly for each participant). Bottom panel: N2pc difference waveforms (ipsilateral minus contralateral ERPs) for dual-feature targets (solid line), and summed N2pc difference waves for the two types of single-feature targets (dashed line) components. These observations suggest that during the early stage of target selection, attention was deployed independently to each of the two target colours, with both colour selection processes generating separate N2pc components that combined additively during the selection of dual-feature targets (see also Töllner, Zehetleitner, Krummenacher, \& Müller, 2011, for related N2pc evidence from a task where participants had to detect colour singletons, orientation singletons, or redundantly defined targets that were singletons in both dimensions). Critically, the subsequent emergence of a superadditive N2pc to dual-feature targets in Experiment 2 mirrors the superadditive target N2pc found in Experiment 1, despite the fact that objects with two or one target colours now both required a response. This strongly suggests that this superadditivity is not the result of a disengagement of attention from partially target-matching objects that have to be ignored. Instead, the onset of a superadditive $\mathrm{N} 2 \mathrm{pc}$ to dual-feature targets in Experiment 2 is likely to mark the point in time at which feature-based selection processes cease to operate independently, and attentional control processes become sensitive to the joint presence of targetdefining features in the same object.

It could be argued that the similar pattern of N2pc components observed in Experiments 1 and 2 was due to the fact that there were no systematic differences in the attentional task sets adopted by the participants in these two experiments. As in Experiment 1, they may have primarily searched for objects with both target colours in Experiment 2. The observation that RTs were faster for dual-feature compared with single-feature targets is in line with this interpretation. However, and in contrast to Experiment 1, single-feature target objects always required a manual response in Experiment 2, and these objects were never accompanied by an object with both target colours in the same display. For these reasons, participants in Experiment 2 had no incentive to rapidly withdraw attention from single-feature targets. The superadditivity of the dualtarget N2pc observed in this experiment can therefore not be attributed to such attentional disengagement processes. It should also be noted that behavioural performance and the temporal pattern of N2pc components in Experiment 2 were entirely unaffected by whether each of the two task-relevant colours always occupied the same relative position within search display objects or whether these colour-position mappings were variable. This suggests that information about the spatial configuration of target colours has little if any impact on attentional guidance processes during colour/colour conjunction search (see also Berggren \& Eimer, in press).

\section{General discussion}

The goal of the present study was to investigate the time course of attentional selection processes that are activated in search tasks where targets are defined by the conjunction of two features within the same dimension (colour). Previous behavioural experiments have found that this type of search is generally very difficult (Wolfe et al., 1990; Wing \& Allport, 1972). However, other studies have suggested that colour/ colour conjunction search can sometimes operate in a relatively efficient fashion (Carrasco et al., 1998; Linnell \& Hymphreys, 2001, 2002). We employed the N2pc component as a temporal marker of the deployment of attention to colour/ 
colour conjunction targets and to objects that matched one target-defining colour. Experiment 1 showed that attention was initially allocated in parallel and independently to all objects with target-matching colours. At a later stage that was marked by the emergence of a superadditive N2pc component to target objects, attentional selection processes no longer operated in an independent feature-specific fashion, suggesting that at this stage, information about the presence of multiple target-defining attributes of the same object was combined across features. Experiment 2 ruled out an alternative interpretation of the superadditive target $\mathrm{N} 2 \mathrm{pc}$ in terms of a selective withdrawal of attention from partially target-matching distractor objects.

Several aspects of these N2pc results appear inconsistent with the account of attentional guidance mechanisms during colour/colour conjunction search that was postulated by the Guided Search model (Wolfe, 2007). First, and most fundamentally, Guided Search proposes a hybrid serial-parallel model of attentional processing, where individual objects are selected serially, but can be processed in parallel during a subsequent object recognition stage (Moore \& Wolfe, 2001). The observation that $\mathrm{N} 2 \mathrm{pc}$ components were triggered by partially target-matching distractors in the competition trials of Experiment 1 where these objects were accompanied by a target on the vertical meridian, and the fact that the sum of these N2pcs to partially matching distractors were initially similar in size to the target N2pc, are not in line with the hypothesis that feature-guided selection operates in a strictly serial fashion. These findings suggest instead that attention was initially deployed in parallel to all objects with targetmatching colours in the same display (see also Eimer \& Grubert, 2014a, for analogous N2pc results indicative of parallel selection during colour/shape conjunction search). It should be noted that the N2pc to partially matching nontargets in Experiment 1 tended to be larger on no-competition relative to competition trials. This observation suggests that the simultaneous selection of two objects with target-matching colours on competition trials may not have been entirely independent, as assumed by a strong version of a parallel selection model. This tendency towards attenuated N2pcs on these trials may have been due to competitive interactions between two concurrent attentional selection processes. Thus, while the pattern of N2pc results observed on competition trials shows that attention was allocated temporally in parallel to two targetcolour objects, it does not provide unequivocal evidence that these two selection processes operated in a fully independent fashion.

The rapid parallel feature-based selection processes found in the present study may be based on the same mechanisms as the enhancements of neural activity for currently attended features that are elicited in a spatially global fashion across the visual field (feature-based attention; Serences \& Boynton, 2007; Eimer, 2014, 2015, for further discussion). In addition, the fact that in both experiments, the N2pc to objects with two target colours was initially equal to the sum of the two N2pc components to objects with one or the other of these colours appears inconsistent with the proposal that attention can only be guided by a single feature within a given dimension at any point in time (Wolfe, 2007). This pattern of N2pc results suggests that both target colours elicited parallel selection processes, triggering separate N2pc components which combined additively during the attentional selection of conjunctively defined target objects (Experiment 1) or dual-feature targets (Experiment 2). If this interpretation was correct, it implies that attention can be guided simultaneously by more than one colour, which would also be in line with behavioural and electrophysiological evidence for multiple-colour attentional task sets (Irons et al., 2012; Grubert \& Eimer, in press; Berggren \& Eimer, in press).

The emergence of a superadditive N2pc to target objects defined by a particular colour/shape conjunction has been interpreted as marking the transition from an early featurebased phase of attentional control to a second object-based phase where the allocation of attention is controlled by an integration of target-defining object features across feature dimensions (Eimer \& Grubert, 2014a). The fact that a very similar temporal pattern of $\mathrm{N} 2 \mathrm{pc}$ components was found in the present study suggests that analogous integration processes also operate for target features from the same dimension. The exact nature of the mechanisms responsible for this type of feature integration remains to be determined. One possibility is that spatially selective biases that are initially triggered in an independent feature-based fashion at overlapping retinotopic locations interact in a facilitatory fashion, resulting in a superadditive bias for objects with multiple targetmatching features (see also Duncan, Humphreys, \& Ward, 1997, for a related "integrated competition" account of attentional object selection). In this case, the superadditive target $\mathrm{N} 2 \mathrm{pc}$ would reflect feature integration in the sense that signals from different task-relevant features of the same object start to interact within or across dimensions. This does not necessarily imply that at this stage features are fully integrated and bound into a single phenomenologically accessible object file (Treisman, 1988). Such fully integrated object representations should specify the features of a particular object and their spatial arrangement within this object in a way that is available to explicit top-down control and object recognition processes. In fact, results from a recent ERP study of colour/colour conjunction search (Berggren \& Eimer, in press) suggest that attentional control processes are remarkably insensitive to colour configuration signals from the same object. When a target defined by a specific spatial arrangement of two colours (e.g., red above green) and a distractor object with the same colours in the reverse spatial configuration (e.g., green above red) appeared on opposite sides of the same search display, no spatially selective modulations of occipito-temporal ERP 
waveforms were elicited at all during the first $500 \mathrm{~ms}$ after search display onset, demonstrating that top-down attentional control mechanisms were unable to differentiate between a target and a task-irrelevant distractor object on the basis of their colour configuration. Such observations strongly suggest that the type of feature integration process that emerges during conjunction search around $250 \mathrm{~ms}$ after the onset of a search display reflects relatively early facilitatory interactions between feature-based attentional biases in visual cortex but not yet the formation of fully explicit representations of the spatial-configural layout of target objects.

Overall, the present study provides new electrophysiological evidence that attentional allocation processes during colour/colour conjunction search initially operate in parallel for different task-relevant colours before they become sensitive to the presence of multiple target colours in the same object. How far can these conclusions be generalised to more typical behavioural conjunction search experiments where display set size is manipulated, RT search slopes are the main dependent variable, and target detection is usually much harder than in the present experiments? In many conjunction search experiments with large set sizes, target-present and target-absent RTs are very slow $(3,000-5,000 \mathrm{~ms}$ or even longer), and participants usually move their eyes repeatedly to different regions of a search display before a target is found (see Hulleman \& Olivers, in press, for a recent argument that eye movements should play a central explanatory role in theories of visual search). With large display set sizes, colour/colour conjunction search is usually hard (Wolfe et al., 1990), whereas it can be relatively efficient when search displays only contain few items (Linnell \& Hymphreys, 2001, 2002). N2pc experiments like the ones reported typically employ search displays with small set sizes to ensure that targets or partially target-matching distractors can be detected and selected within the first selection episode immediately after the presentation of a search display, before any eye movements. The assumption is that the attentional processes during this initial selection period are not qualitatively different from the processes that are elicited in more difficult search tasks during subsequent episodes, where they are initiated repeatedly after each new eye movement brings a different region of a search display into the functional field of view (see Eimer, 2014, for further discussion).

If this is correct, the primary reason that the colour/colour conjunction search was inefficient in the study by Wolfe et al. (1990) may not have been display set size but instead the presence of multiple distractor objects that shared one feature with the target in their experiments. The fact that targets were accompanied by only one distractor with a target-matching colour in the competition trials of the present study is likely to have facilitated a relatively rapid preferential allocation of attention to target objects, as reflected by the emergence of a superadditive target $\mathrm{N} 2 \mathrm{pc}$. If attention was initially allocated in parallel to all objects with target-matching colours, as suggested by the present N2pc results, such a spatially selective attentional bias for target objects may appear substantially later when displays include numerous distractors that share one of the two target-defining colours. To test this prediction, future ERP studies should employ tasks where colour/colour conjunction targets appear together with several distractor objects that all match one of the two target colours. If the simultaneous deployment of attention to multiple objects with target-matching features impairs the selective allocation of spatial attention to target objects, search performance should be strongly impaired, and a superadditive target N2pc should emerge later or not at all under these conditions.

Acknowledgment This work was supported by grant ES/L016400/1 from the Economic and Social Research Council (ESRC), UK.

\section{References}

Berggren, N., \& Eimer, M. (in press). The guidance of spatial attention during visual search for colour combinations and colour configurations. Journal of Experimental Psychology: Human Perception and Performance.

Carlisle, N. B., Arita, J. T., Pardo, D., \& Woodman, G. F. (2011). Attentional templates in visual working memory. Journal of Cognitive Neuroscience, 31, 9315-9322.

Carrasco, M., Ponte, D., Rechea, C., \& Sampedro, M. J. (1998). "Transient structures": The effect of practice and distractor grouping on within-dimension conjunction searches. Perception \& Psychophysics, 60, 1243-1258.

Desimone, R., \& Duncan, J. (1995). Neural mechanisms of selective visual attention. Annual Review of Neuroscience, 18, 193-222.

Duncan, J., \& Humphreys, G. W. (1989). Visual search and stimulus similarity. Psychological Review, 96, 433-458.

Duncan, J., Humphreys, G. W., \& Ward, R. (1997). Competitive brain activity in visual attention. Current Opinion in Neurobiology, 7, 255-261.

Eimer, M. (1996). The N2pc component as an indicator of attentional selectivity. Electroencephalography and Clinical Neurophysiology, 99, 225-234.

Eimer, M. (2014). The neural basis of attentional control in visual search. Trends in Cognitive Sciences, 18, 526-535.

Eimer, M. (2015). EPS Mid-Career Award 2014: The control of attention in visual search: Cognitive and neural mechanisms. The Quarterly Journal of Experimental Psychology, 68, 2437-2463.

Eimer, M., \& Grubert, A. (2014a). The gradual emergence of spatially selective target processing in visual search: From feature-specific to object-based attentional control. Journal of Experimental Psychology: Human Perception and Performance, 40, 1819-1831.

Eimer, M., \& Grubert, A. (2014b). Spatial attention can be allocated rapidly and in parallel to new visual objects. Current Biology, 24, 193-198.

Eimer, M., Kiss, M., \& Nicholas, S. (2011). What top-down task sets do for us: An ERP study on the benefits of advance preparation in visual search. Journal of Experimental Psychology: Human Perception and Performance, 37, 1758-1766.

Folk, C. L., \& Anderson, B. A. (2010). Target-uncertainty effects in attentional capture: Color singleton set of multiple attentional control settings? Psychonomic Bulletin and Review, 17, 421-426. 
Folk, C. L., \& Remington, R. W. (1998). Selectivity in distraction by irrelevant featural singletons: Evidence for two forms of attentional capture. Journal of Experimental Psychology: Human Perception and Performance, 24, 847-858.

Folk, C. L., Remington, R. W., \& Johnston, J. (1992). Involuntary covert orienting is contingent on attentional control settings. Journal of Experimental Psychology: Human Perception and Performance, $18,1030-1044$.

Grubert, A., \& Eimer, M. (2016). The speed of serial attention shifts in visual search: Evidence from the N2pc component. Journal of Cognitive Neuroscience, 28, 319-332.

Grubert, A., \& Eimer, M. (in press). All set, indeed! N2pc components reveal simultaneous attentional control settings for multiple target colours. Journal of Experimental Psychology: Human Perception and Performance.

Hickey, C., McDonald, J. J., \& Theeuwes, J. (2006). Electrophysiological evidence of the capture of visual attention. Journal of Cognitive Neuroscience, 18, 604-613.

Hulleman, J., \& Olivers, C.N. (in press). The impending demise of the item in visual search. Behavioral and Brain Sciences.

Irons, J. L., Folk, C. L., \& Remington, R. W. (2012). All set! Evidence of simultaneous attentional control settings for multiple target colors. Journal of Experimental Psychology: Human Perception and Performance, 38, 758-775.

Kiss, M., Grubert, A., \& Eimer, M. (2013). Top-down task sets for combined features: Behavioral and electrophysiological evidence for two stages in attentional object selection. Attention, Perception, \& Psychophysics, 75(2), 216-228.

Linnell, K. J., \& Humphreys, G. W. (2002). Visual search within and across dimensions: A case for within-dimension grouping. British Journal of Psychology, 93, 115-135.

Linnell, K. J., \& Hymphreys, G. W. (2001). Spatially parallel processing of within-dimension conjunctions. Perception, 30, 49-60.

Luck, S. J., \& Hillyard, S. A. (1994). Spatial filtering during visual search: Evidence for human electrophysiology. Journal of Experimental Psychology: Human Perception and Performance, 20, 1000-1014.

Miller, J., Patterson, T., \& Ulrich, R. (1998). Jackknife-based method for measuring LRP onset latency differences. Psychophysiology, 35, 99-115.

Moore, C. M., \& Wolfe, J. M. (2001). Getting beyond the serial/parallel debate in visual search: A hybrid approach. In K. Shapiro (Ed.), The limits of attention: Temporal constraints on human information processing (pp. 178-198). Oxford: Oxford University Press.

Müller, H. J., Heller, D., \& Ziegler, J. (1995). Visual search for singleton feature targets within and across feature dimensions. Perception \& Psychophysics, 57, 1-17.

Serences, J. T., \& Boynton, G. M. (2007). Feature-based attentional modulations in the absence of direct visual stimulation. Neuron, 55, 301-312.

Shibata, K., Yamagishi, N., Goda, N., Yoshioka, T., Yamashita, O., Sato, M. A., \& Kawato, M. (2008). The effects of feature attention on prestimulus cortical activity in the human visual system. Cerebral Cortex, 18, 1664-1675.

Töllner, T., Gramann, K., Müller, H. J., Kiss, M., \& Eimer, M. (2008). Electrophysiological markers of visual dimension changes and response changes. Journal of Experimental Psychology: Human Perception and Performance, 34, 531-542.

Töllner, T., Zehetleitner, M., Krummenacher, J., \& Müller, H. J. (2011). Perceptual basis of redundancy gains in visual pop-out search. Journal of Cognitive Neuroscience, 23, 137-150.

Treisman, A. M. (1988). Features and objects: The fourteenth Bartlett memorial lecture. The Quarterly Journal of Experimental Psychology, 40, 201-237.

Treisman, A. M., \& Gelade, G. (1980). A feature-integration theory of attention. Cognitive Psychology, 12, 97-136.

Wing, A., \& Allport, D. A. (1972). Multidimensional encoding of visual form. Perception \& Psychophysics, 12, 474-476.

Wolfe, J. M. (1994). Guided Search 2.0: A revised model of visual search. Psychonomic Bulletin and Review, 1, 202-238.

Wolfe, J. M. (2007). Guided Search 4.0: Current progress with a model of visual search. In W. Gray (Ed.), Integrated models of cognitive systems (pp. 99-119). New York, NY: Oxford University Press.

Wolfe, J. M., Yu, K. P., Stewart, M. I., Shorter, A. D., Friedman-Hill, S. R., \& Cave, K. R. (1990). Limitations on the parallel guidance of visual search: Color $\mathrm{x}$ color and orientation $\mathrm{x}$ orientation conjunctions. Journal of Experimental Psychology: Human Perception and Performance, 16, 879-892.

Woodman, G. F., \& Luck, S. J. (1999). Electrophysiological measurement of rapid shifts of attention during visual search. Nature, 400, $867-869$.

Woodman, G. F., \& Luck, S. J. (2003). Serial deployment of attention during visual search. Journal of Experimental Psychology: Human Perception and Performance, 29, 121-138. 